06

\title{
Исследование вакансионной системы реструктурированного цинка методом аннигиляции позитронов
}

\author{
(С) Е.М. Соловьев, ${ }^{1}$ Б.В. Спицын, ${ }^{2,}$ Р.С. Лаптев, ${ }^{3}$ А.М. Лидер, ${ }^{3}$ Ю.С. Бордулев, ${ }^{3}$ А.А. Михайлов ${ }^{3}$ \\ ${ }^{1}$ НИЦ Специальные технологии, \\ 127238 Москва, Россия \\ ${ }^{2}$ Институт ффизической химии и электрохимии им. А.Н. Фрумкина РАН, \\ 119071 Москва, Россия \\ ${ }^{3}$ Национальный исследовательский Томский политехнический университет, \\ 634050 Томск, Россия \\ ฯ e-mail: spitsyn@phyche.ac.ru
}

(Поступило в Редакцию 23 января 2017 г.)

Получен реструктурированный цинк (цинк высокой чистоты, подготовленный путем механо-термического воздействия различного уровня интенсивности), и проведено изучение его структуры методами позитронной спектроскопии. В реструктурированных образцах обнаружены и количественно исследованы объемные дефекты вакансионного типа (вакансии и вакансионные кластеры), а также области с расширенной кристаллической решеткой. Эти эффекты являются причиной возникновения внутренних микронапряжений в материале, которые обуславливают изменение свойств материала.

DOI: 10.21883/JTF.2018.06.46017.2173

\section{Введение}

Сочетание механического и термического воздействий на расплавленный металл позволяет значительно улучшить чистоту металла, а также существенно изменить его структуру и свойства [1-4].

Основа метода состоит в специальной обработке расплавленного металла в контролируемой газовой среде (вакуум, инертная или восстановительная атмосфера) в условиях переменных в пространстве и во времени массы обрабатываемого материала, температуры и давления. Изменяя указанные параметры, представляется возможным изменять как химический состав обрабатываемого материала, так и его внутреннюю структуру.

Предварительными опытами было показано, что у чистых металлов можно изменять кристаллическое строение, механические модули, электрохимический потенциал, параметры сверхпроводимости и другие структурночувствительные свойства [5-8].

Подобные возможности в настоящее время востребованы не только для повышения чистоты и свойств легкоплавких металлов (свинец, цинк, алюминий), но и, в особенности, для тугоплавких металлов (платина, цирконий, гафний, вольфрам). Перспективными направлениями последующих научно-исследовательских работ представляются аналогичные научные и технологические исследования металлов и сплавов на основе железа, меди и других металлов.

Поскольку появление и сохранение особых свойств наблюдается как в чистых металлах, так и в их сплавах (равновесных, неравновесных), следует отметить, что разрабатываемая новая технология структурирования металлических материалов, при ее масштабировании будет экономически эффективной и будет удовлетворять современным экологическим требованиям.

Механотермическая обработка различного уровня интенсивности оказывает влияние на электронную структуру материала $[9,10]$. Соответственно имеются основания полагать, что изменения в электронной, а также дефектной структуре после обработки оказывают влияние на изменение времени жизни позитронов в материале. В связи с этим в настоящей работе был применен метод спектроскопии времени жизни позитронов, обладающий высокой чувствительностью к подобным изменениям.

Таким образом, цель настоящей работы состояла в получении реструктурированного цинка и изучении строения его методами позитронной спектроскопии.

\section{Материалы и методы исследования}

В исследованиях были использованы образцы реструктурированного цинка. Кристаллизация металлического расплава с заданной массой $(G)$ и первоначальной температурой $\left(T_{0}\right)$ производилась при его объемном переохлаждении в нестационарных условиях и заданных во времени режимах воздействия поля центробежных сил, определяемого центрифугированием, а также температурно-тепловым воздействием на расплав контактирующим с ним газовым теплоносителем с заданными во времени характеристиками (температурой и расходом).

Скорость охлаждения материала устанавливалась в интервале от 5 до $100 \mathrm{deg} / \mathrm{s}$. Форма материала в процессе обработки представляла собой вращающийся вокруг своей оси цилиндр, в полость которого с заданными во времени значениями расхода и температуры вводился 


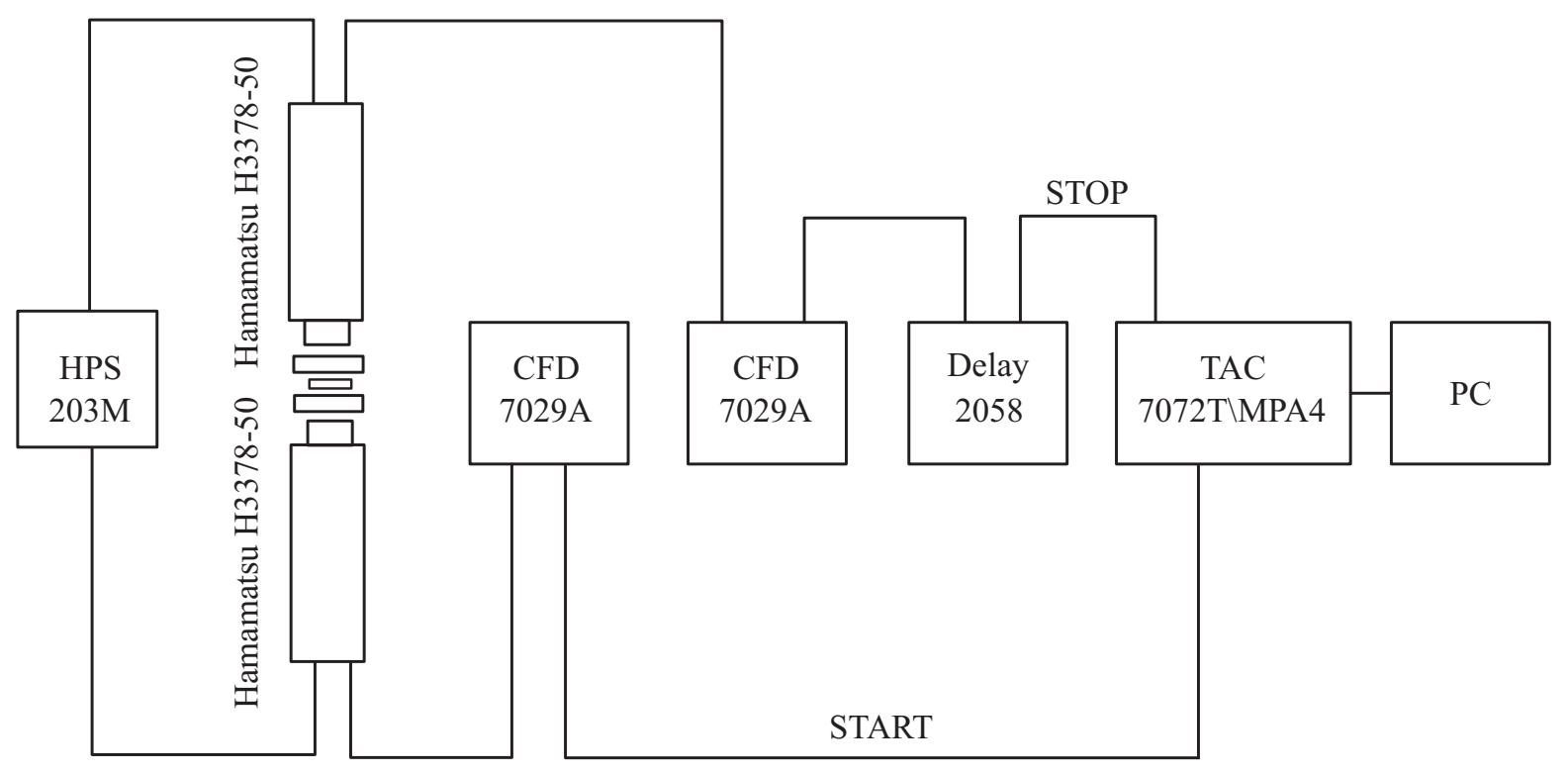

Схема спектрометра для исследования временного распределения аннигиляции позитронов.

горячий газовый агент. Коэффициент перегрузки обеспечивался в диапазоне от 200 до $1000 \mathrm{~g}$. Масса материала, обработанного таким образом, может составлять от 50 до $150 \mathrm{~kg}$ и более. Преобразованный и сформированный таким образом материал существенно изменяет свою структуру с образованием новых дефектов, что, повидимому, изменяет электронную структуру материала в целом. Степень обработки исследованных образцов варьировалась. По степени интенсивности обработки образцы условно обозначены Zn0 (без обработки), Zn1, $\mathrm{Zn} 2, \mathrm{Zn3}$. Чем выше номер образца тем выше степень его обработки. Для исключения влияния особой структуры приповерхностных слоев образцы подвергались механохимической обработке в кислотной среде.

В качестве инструмента исследования был использован метод спектроскопии времени жизни позитронов, который подтвердил свою эффективность в исследовании электронной структуры твердых тел [11-15]. Спектрометр времени жизни позитронов был создан на кафедре общей физики Физико-технического института Томского политехнического университета. В качестве источника позитронов использовался изотоп ${ }^{44} \mathrm{Ti}$ с максимальной энергией позитронов $1.467 \mathrm{MeV}$ и активностью $1.26 \mathrm{MBq}$. Спектрометр реализован на основе „быстро-быстрой“ схемы. Для определения времени жизни позитронов в веществе использовался метод запаздывающих совпадений. Суть метода заключается в измерении скорости счета запаздывающих совпадений между ядерным $\gamma$-квантом с энергией $1.157 \mathrm{MeV}$ (сигнал „старт“), который испускается почти одновременно с позитроном, и аннигиляционным $\gamma$-квантом с энергией $0.511 \mathrm{MeV}$ (сигнал „стоп“).

Регистрация $\gamma$-квантов осуществляется сцинтилляционными детекторами Hamamatsu H3378-50 на основе кристаллов $\mathrm{BaF} 2$ цилиндрической формы диа- метром $30 \mathrm{~mm}$ и толщиной $25 \mathrm{~mm}$. Питание детекторов осуществляется с помощью высоковольтного источника питания (HPS, NHQ 203M). Спектрометрический тракт включает в себя два дифференциальных дискриминатора постоянной составляющей (CFD, FAST ComTech 7029A), блок наносекундной задержки (DELAY, Canberra 2058), время-цифровой преобразователь (TAC, FAST ComTech 7072T), многопараметровый многоканальный анализатор (MPA4) и персональный компьютер с программным обеспечением для набора спектров (РС). Схема спектрометра для исследования временного распределения аннигиляции позитронов представлена на рисунке.

Временно́е разрешение спектрометра с использованием изотопа ${ }^{44} \mathrm{Ti}$ составляет $240 \mathrm{ps}$, скорость счета 130 counts/s. Обработка спектров осуществлялась с помощью программного обеспечения LT 10 [16]. Для исследования времени жизни позитронов было набрано по 2 спектра, не менее $5 \cdot 10^{6}$ событий в каждом спектре.

Для проведения эксперимента образцы упаковывались в так называемой „сэндвич-геометрии“, согласно которой 2 образца от каждой серии располагались с двух сторон от источника позитронов. Такая геометрия обеспечивает условие попадания максимальной доли излучаемых позитронов в исследуемый материал.

\section{Результаты и обсуждение}

Для разложения спектров времени жизни (СВЖ) позитронов в исследуемых образцах помимо анализа среднего времени жизни позитронов в материале была использована двухкомпонентная модель захвата позитронов дефектами (two state trapping model) [17]. Спектры математически раскладывались на две экспоненциальные составляющие, характеризуемые временем 
Результаты анализа СВЖ позитронов в образцах цинка

\begin{tabular}{c|c|c|c|c|c|c|c}
\hline \multirow{2}{*}{ Образец } & \multirow{2}{*}{$\begin{array}{c}\text { Среднее время жизни } \\
\text { позитронов в образце }\left(\tau_{\text {mean }}, \mathrm{ps}\right)\end{array}$} & \multicolumn{4}{|c}{ Двухкомпонентная модель захвата позитронов дефектами } \\
\cline { 3 - 7 } & & 1 компонента & \multicolumn{4}{|c}{2 компонента } \\
\cline { 3 - 7 } & & $\tau_{1}, \mathrm{ps}$ & $I_{1}$ & $\tau_{2}, \mathrm{ps}$ & $R, \mathrm{~A}$ & $V$, вакансий & $I_{2}$ \\
\hline 0 & 144 & 144 & 100 & - & - & - & - \\
Zn1 & 152 & 149 & 78 & 250 & 1.8 & 1.6 & 9 \\
Zn2 & 155 & 150 & 76 & 237 & 1.7 & 1.4 & 21 \\
Zn3 & 169 & 160 & 67 & 267 & 1.9 & 2 & 23
\end{tabular}

жизни позитронов $(\tau)$ и интенсивностью компоненты $(I)$. Каждая компонента соответствует определенному состоянию позитрона в материале: делокализованное состояние позитрона в решетке $\left(\tau_{1}\right)$ и локализованное состояние в дефекте $\left(\tau_{2}\right)$. Результаты обработки спектров представлены в таблице.

Среднее время жизни является наиболее робастным параметром и не зависит от модели обработки. По величине среднего времени жизни позитронов можно судить об общей дефектности исследуемого материала. Среднее время жизни позитронов в условно бездефектном образце $\mathrm{Zn0}$ хорошо согласуется с литературными данными времени жизни позитронов в бездефектной решетке цинка -148 ps [18]. Из значений среднего времени жизни также видно, что с номером образца оно увеличивается, что говорит о том, что изменяется средняя электронная плотность (уменьшается), что свидетельствует об увеличении дефектности материала.

Двухкомпонентная обработка показала, что во всех спектрах, кроме спектра $\mathrm{Zn0}$, имеются короткоживущая и долгоживущая компоненты. Время жизни короткоживущей компоненты свидетельствует о том, что эта компонента связана с аннигиляцией позитрона в решетке материала. Изменение значения данной компоненты указывает на изменение электронной плотности в решетке кристалла (бездефектная область). Из таблицы видно, что значение времени жизни данной компоненты увеличивается, что свидетельствует о расширении кристаллической решетки. Также можно заметить, что значение данной компоненты тем больше, чем выше номер образца. Ее значение выходит на свое максимальное значение для образца $\mathrm{Zn} 3$.

Вторая компонента - долгоживущая, связанная с захватом позитронов дефектами. Ее нет в образце Zn0, что говорит о том, что в этом образце отсутствуют дефекты, захватывающие позитроны. Время жизни данной компоненты изменяется от образца к образцу, что говорит о различном типе и размере дефектов, присутствующих в материале. Время жизни долгоживущей компоненты в образце Zn1 составляет 250 ps, что на 30 ps выше экспериментального значения времени жизни позитрона в вакансии цинка [19]. Это может говорить о том, что в Zn1 имеются дефекты в форме вакансионных кластеров. Время жизни данной компоненты в образце $\mathrm{Zn} 1$ ненамного снижается $(237 \mathrm{ps})$, что говорит об уменьшении их объема. В образце Zn2 время жизни этой компоненты достигает своего максимума, что может свидетельствовать о росте размера кластеров. В образце Zn3 мы видим небольшое снижение времени жизни данной компоненты.

Из таблицы видно, что с увеличением номера образца увеличивается и выходит на плато интенсивность $I_{2}$ дефектной компоненты. Это говорит о росте концентрации вакансионных кластеров с увеличением степени обработки. В целом по данным СВЖ позитронов видно, что образец 3 мало отличается от образца 2.

Расчет размера кластеров производился по полуэмпирической модели, предложенной Е.П. Прокопьевым и В.И. Графутиным в работе [20]. При проведении расчетов учитываются следующие упрощения:

- полость, в которой локализуется позитрон, аппроксимируется сферической потенциальной ямой радиуса $R_{0}$ с бесконечно высоким потенциальным барьером;

- волновая функция позитрона на стенах сферы обращается в нуль;

- аннигиляция позитронов происходит на внешних электронах среды, образующих стенку полости с толщиной электронного слоя $\Delta \mathrm{R}$.

Таким образом, зависимость времени жизни позитрона от размера поры можно будет выразить в виде:

$$
\tau_{d}=\tau_{0}\left|1-\frac{R}{R+\Delta R}+\frac{1}{2 \pi} \sin \left(\frac{2 \pi R}{R+\Delta R}\right)\right|^{-1},
$$

где радиус поры $R=R_{0}-\Delta R, \tau_{d}$ и $\tau_{0}$ - времена жизни позитрона в дефекте и в решетке соответственно.

Считая, что моновакансия образуется одним удаленным атомом и имеет размер, равный размеру ячейки Вигнера-Зейтца, можно определить значение $\frac{R}{R+\Delta R}$ для моновакансии цинка. Используя справочные данные [11], находим объем ячейки Вигнера-Зейтца, приходящийся на один атом:

$$
V_{\mathrm{wsc}}=\frac{M[\mathrm{~g} / \mathrm{mol}]}{\rho\left[\mathrm{g} / \mathrm{cm}^{3}\right] N_{A}\left[\mathrm{~mol}^{-1}\right]}=1.522 \cdot 10^{-23} \mathrm{~cm}^{3},
$$

где $M$ - атомная масса цинка, $\rho$ - плотность цинка, $N_{a}$ - число Авогадро. Найдем радиус сферы $R$, имеющую объем ячейки Вигнера-Зейтца:

$$
R=\sqrt[3]{\frac{3^{*} V_{\mathrm{wsc}}}{4^{*} \pi}}=1.537 \cdot 10^{-8} \mathrm{~cm}
$$


Время жизни позитронов в бездефектной решетке цинка $\tau_{0}$ и в моновакансии $\tau_{v}$ составляет $\sim 144$ и $220 \mathrm{ps}$ соответственно [17]. Подставляя данные значения в выражение (1), определили значение $\frac{R}{R+\Delta R} \approx 0.421$, отсюда толщина электронного слоя $\Delta R=2.114 \AA$.

Используя выражение (1) и считая, что толщина электронного слоя не зависит от размера вакансионного комплекса, а также, что объем комплекса, состоящего из $m$ моновакансий, равен сумме объемов $m$ моновакансий, в настоящей работе был рассчитан радиус свободного объема кластеров, а также объем кластеров (в вакансиях) в исследованных образцах. Результаты представлены в таблице. Из рассчитанных результатов видно, что почти во всех случаях объем кластера больше объема одной вакансии, но меньше объема двух вакансий. Это говорит о том, что в реструктурированных образцах наблюдается смесь моновакансий и дивакансий. В образце, обозначенном как Zn3, моновакансий практически нет, весь свободный объем составляют дивакансии.

\section{Заключение}

В настоящей работе показано, что указанное механотермическое воздействие на материал существенно влияет на дефектную структуру материала, что сказывается на характере поведения позитронов в исследуемом материале. Во-первых, данное воздействие способствует расширению кристаллической решетки. Расширение кристаллической решетки увеличивается с увеличением степени деформации. Помимо этого, в образцах возникают объемные дефекты вакансионного типа (моно- и дивакансии). С увеличением степени воздействия происходит рост объема дефектов и количество дивакансий начинает оказывать преобладающий эффект на захват позитронов. На протяжении всего эксперимента наблюдается увеличение концентрации дефектов (с выходом на плато) с увеличением степени обработки материала.

\section{Список литературы}

[1] Vrancken B., Thijs L., Kruth J.P., van Humbeeck J. // J. Alloys and Compounds. 2012. Vol. 541. P. 177-185.

[2] Santos E., Abe F., Kitamura Y., Osakada K., Shiomi M. // 13rd Annual Intern. Sol. Freeform Fabrication Symposium. Austin, TX, 2002. P. 180-186.

[3] Xue M., Heichal Y., Chandra S., Mostaghimi J. // J. Mater. Sci. 2007. Vol. 42. N 1. P. 9-18.

[4] Ratle A., Pandolfelli Vol. C., Allaire C., Rigaud M. // British Ceramic Transactions. 1997. Vol. 96. N 6. P. 225-230.

[5] Edalati K., Fujioka T., Horita Z. // Mater. Sci. Engineer. A. 2008. Vol. 497. N 1. P. $168-173$.

[6] Pugh S.F. // The London, Edinburgh and Dublin Philosophical Magazine and Journal of Science. 1954. Vol. 45. N 367. P. 823-843.

[7] Hollingsworth M.D. // Science. 2002. Vol. 295. N 5564. P. $2410-2413$.
[8] Finnemore D.K., Stromberg T.F., Swenson C.A. // Phys. Rev. 1966. Vol. 149. N 1. P. 231.

[9] Brudnyi V.N., Kosobutsky A.V., Kolin N.G. // Phys. Sol. St. 2011. Vol. 53. N 4. P. 679-688.

[10] Песин Л.А., Грибов И.В., Москвина Н.А., Кузнещов В.Л., Евсюков С.Е., Богатырева М.Е., Хананова А.В. // Вестн. Южно-Уральского гос. ун-та. Сер. Математика. Механика. Физика. 2011. № 32. С. 249.

[11] Bordulev Y.S., Laptev R.S., Kudiyarov V.N., Lider A.M. // Adv. Mater. Res. 2014. N 880. P. 93-100.

[12] Laptev R.S., Bordulev Y.S., Kudiyarov V.N., Lider A.M., Garanin G.V. // Adv. Mater. Res. 2014. N 880. P. 134-140.

[13] Kuznetsov P.V., Mironov Y.P., Tolmachev A.I., Rakhmatulina T.V., Bordulev Y.S., Laptev R.S., Lider A.M., Mikhaylov A.A., Korznikov A.V. // AIP Conf. Proc. 2014. N 1623. P. 327-330.

[14] Laptev R.S., Lider A.M., Bordulev Y.S., Kudiiarov V.N., Gvozdyakov D.V. // Key Engineering Materials. Trans Tech. Publications. 2016. Vol. 683. P. 256-261.

[15] Kuznetsov P., Cizek J., Hruska P., Anwad W., Bordulev Yu., Lider A., Laptev R., Mironov Yu. // Adv. Mater. with Hierarchical Structure for new Technoloies and Reliable Structures. AIP Publishing. 2015. Vol. 1683. P. 020116.

[16] Giebel D., Kansy J. // Phys. Proc. 2012. T. 35. P. 122-127.

[17] Hautojärvi P., Corbel C. In: Proceedings of the International School of Physics „Enrico Fermi“", Course CXXV / Ed. A. Dupasquier, A.P. Mills. IOS Press, Varenna, 1995. P. 1491.

[18] Carlos Hidalgo, Soren Linderoth. // Phys. Rev. B. 1987. Vol. 36. N 13. P. 36.

[19] Campillo J.M., Plazaola F., De Diego N. // J. Phys.: Condens. Matter. 2000. Vol. 12. N 46. P. 9715.

[20] Бритков О.М., Гаврилов С.А., Графутин В.И., Дягилев В.В., Калугин В.В., Илюхина О.В., Фунтиков Ю.В. // Вопросы атомной науки и техники. Сер. Теор. и прикл. физика. 2004. № 3. С. 40-50. 